\title{
Balance energético en el primer ciclo de corta de tres especies de eucalipto con diferentes niveles de manejo en el secano Mediterráneo de Chile central
}

\author{
Energy balance in the first cutting cycle of three eucalyptus species \\ with different levels of management in the Mediterranean drylands of central Chile
}

\begin{abstract}
Antonio Cabrera-Ariza ${ }^{\text {a*, }}$, Rómulo Santelices-Moya a, Sergio Espinoza-Meza a , Carlos Magni-Díaz ${ }^{\text {b }}$
*Autor de correspondencia: a Universidad Católica del Maule, Facultad de Ciencias Agrarias y Forestales, Centro de Desarrollo para el Secano Interior, Avenida San Miguel 3605, Talca, Chile, tel.: 56971 2413728, acabrera@ucm.cl

${ }^{b}$ Universidad de Chile, Facultad de Ciencias Forestales y de Conservación de la Naturaleza, Santiago de Chile, Chile.
\end{abstract}

\begin{abstract}
SUMMARY
The Mediterranean Secano of central Chile is a degraded zone, with high rates of erosion and a very fluctuating climate, with long periods of low rainfall, which limits its productivity. An alternative for this area is the cultivation of energy-bound eucalyptus species. The objective of this research was to evaluate the energy balance at the field level in the first rotation ( 3 years) of three eucalyptus species, comparing basic and intensive management. For this purpose, the species Eucalyptus camaldulensis, Eucalyptus tereticornis and Eucalyptus globulus were established with two different levels of management, one basic (20 cm deep subsoiling, planting and harvesting) and another intensive (50 cm deep plowing, subsoiling, application of herbicide, planting, fertilization and harvesting). Results show that for both types of management the energy balance is positive, being higher for intensive management. Values ranged between 102,900 and 137,890 $\mathrm{MJ} \mathrm{ha}^{-1}$ year $^{-1}$ in the basic management and 125,730 and 160,050 $\mathrm{MJ}^{-1} \mathrm{year}^{-1}$ in intensive management, depending in both cases on the species. In conclusion, energy plantations with eucalyptus species have a positive energy balance and are a viable alternative for drylands areas, especially the Eucalyptus camaldulensis species.
\end{abstract}

Key words: biomass, energy, basic management, intensive management.

\section{RESUMEN}

El Secano Mediterráneo de Chile central es una zona afectada por altas tasas de erosión y un clima muy fluctuante, con largos períodos de bajas precipitaciones, los que limita su productividad. Una alternativa para esta zona es el cultivo de especies del género Eucalyptus spp. con fines energéticos. El objetivo de esta investigación fue evaluar el balance energético a pie de campo en la primera rotación (3 años) de tres especies de eucalipto, comparando un manejo básico y un manejo intensivo. Para ello, se seleccionaron las especies Eucalyptus camaldulensis, Eucalyptus tereticornis y Eucalyptus globulus, que fueron establecidas con dos diferentes niveles de manejo, uno básico con subsolado a $20 \mathrm{~cm}$, plantación y cosecha y otro intensivo con subsolado a $50 \mathrm{~cm}$, camellón, arado, aplicación de herbicida, plantación, fertilización y cosecha. Los resultados mostraron que para ambos tipos de manejo el balance energético fue positivo, siendo superior la productividad de biomasa y su potencial energético en el caso del manejo intensivo, con valores que variaron entre 102.900 y $137.890 \mathrm{MJ} \mathrm{ha}^{-1}$ versus el manejo básico con valores entre 125.730 y $160.050 \mathrm{MJ} \mathrm{ha}^{-1}$, dependiendo en ambos casos de la especie. A modo de conclusión, las plantaciones energéticas con las tres especies del género Eucalyptus tienen un balance energético positivo y son una alternativa viable para zonas de secano, especialmente Eucalyptus camaldulensis.

Palabras clave: biomasa, energía, manejo básico, manejo intensivo.

\section{INTRODUCCIÓN}

El Secano Mediterráneo de Chile, y en particular el de la región del Maule, con una superficie de más de 540.000 hectáreas, se caracteriza por exhibir condiciones de sitio que resultan marginales para los cultivos tradicionales, tanto agrícolas como forestales. En esta vasta área, el $51 \%$ de los suelos tiene algún grado de erosión (Santelices et al. 2015) y los cultivos presentan rendimientos muy inferiores a los valores óptimos (Novoa 1989). En este contexto, una posible alternativa para parte de estas 540.000 ha, las que presentan condiciones de pendiente (menor del $20 \%$ ) y suelo adecuado, es el cultivo de especies de eucalipto con destinación energética. Los cultivos lignocelulósicos pueden ser utilizados para producir calor y electricidad a través de la combustión directa o la producción de biofuel o biogás a través de la pirólisis y gasificación (Sevigne et al. 2011). La energía proveniente de la biomasa, tanto lignocelulósica como herbácea, se presenta como una alternativa energética sostenible, de hecho, se considera que 
esta energía no tiene emisiones de carbono, ya que el carbono emitido en la combustión es el mismo que las plantas absorben mientras crecen, además, el sistema radical, parte de las hojas que caen y los desechos de la cosecha que quedan en terreno son un aporte de carbono al medio.

Un elemento importante a destacar en el uso de cultivos lignocelulósicos para la producción de energía es la evaluación de la sostenibilidad ambiental de dichos cultivos a largo plazo (Bonari et al. 1992). A tal propósito, el balance energético, representa un instrumento adecuado para la evaluación de dicha sostenibilidad, no se puede gastar más energía en el proceso de la que potencialmente se va a generar. El balance energético pone de manifiesto la energía producida por unidad de energía empleada en el proceso de producción y transformación de la biomasa en energía eléctrica (Scholz y Ellerbrock 2002). Dicho balance ha sido ampliamente usado en diferentes investigaciones (Bonari 1992, Boehmel et al. 2008, Cabrera et al. 2014), obteniendo diferentes resultados dependiendo de la especie y los medios analizados. Sin embargo, existe poca información sobre el particular para especies del género Eucalyptus.

Los valores de producción de biomasa de los Eucalyptus spp. varían en función de la especie, la densidad de plantación, el turno de corta, el manejo y el sitio de plantación, entre otros factores. Generalmente, se obtienen mejores producciones en buenos sitios, con un manejo intensivo del suelo (subsolado profundo) y fertilización. En literatura se han reportado valores que varían desde $1 \mathrm{Mg}$ ha $^{-1}$ año $^{-1}$ hasta $30 \mathrm{Mg} \mathrm{ha}^{-1}$ año-1 (Cromer et al. 1976, Wise y Pittman 1981, Tewari 1992, Foroughbakhch et al. 1997, Van den Broek et al. 2001, Sudha et al. 2003)

En Chile, las plantaciones de Eucalyptus spp. abarcan una superficie de 836.400 hectáreas y son manejadas principalmente con fines pulpables. La principal especie plantada es Eucalyptus globulus Labil. y existen solo plantaciones menores con las especies Eucalyptus nitens Maiden y Eucalyptus camaldulensis Dehnh (INFOR 2017).

A pesar de que existe demanda por bioenergía a base de biomasa (Objetivo 2020 del gobierno), no existen plantaciones a escala dedicadas a la obtención de biomasa para energía. De la misma manera, no existen estudios orientados a la estimación del balance energético ni del potencial de uso en terrenos de secano abandonados o sin uso productivo. Por otro lado, uno de los principales problemas del Secano Interior son los suelos. Generalmente son pobres en materia orgánica con una calidad de sitio que es limitada por bajas precipitaciones, agravado por suelos delgados o de estructura cerrada, cuya retención de agua es baja. Además, producto del pastoreo con ganado bovino tienden a ser suelos compactados. En este sentido, la fertilización y algún tipo de tratamiento al suelo, como subsolado o arado, favorecerán el crecimiento de la planta permitiendo que las raíces tengan una mayor facilidad a la hora de alcanzar la napa freática en los suelos en los que esta esté presente. Por ello, el objetivo del trabajo es evaluar el balance energético a pie de campo en la primera rotación de tres especies de Eucalyptus, comparando un manejo básico y un manejo intensivo (fertilización y tratamiento al suelo), planteando la hipótesis de que una mayor inversión energética en el manejo (manejo intensivo) se verá reflejada en la mejora de las condiciones de sitio y a su vez en una mayor producción de biomasa y, por lo tanto, el balance energético será mayor que en el caso de un manejo básico de la plantación.

\section{MÉTODOS}

Área de estudio. El ensayo se desarrolló en un sitio en la localidad de Huerta Maule, comuna de San Javier, región del Maule, coordenadas geográficas 35³8'26,02" S 72 $02^{\prime} 33,35^{\prime \prime} \mathrm{O}$, presentando características climáticas con precipitaciones medias anuales en el rango de 696$926 \mathrm{~mm}$ año ${ }^{-1}$ (Santibáñez y Uribe 1993), concentradas principalmente en los meses de invierno. En el cuadro 1 se muestran los datos del análisis de suelo realizado en el Centro Tecnológico de Suelos y Cultivos de la Universidad de Talca. Dicho suelo, de acuerdo a Casanova et al. (2007), es un Cambisol flúvi-ferrálico, formados bajo condiciones de humedad excesiva a partir de materiales graníticos. Los análisis del suelo mostraron que la textura del suelo es arcillosa y destacan los bajos niveles nutricionales de nitrógeno, fósforo, potasio, zinc, boro, calcio y sodio. Previo al establecimiento de la plantación, el sitio estaba ocupado por una estepa de Acacia caven (Mol.) Molina de baja densidad ( $\sim 5-8 \%$ de cobertura).

Diseño experimental. La parcela, de una superficie total de 5 ha con la misma exposición y pendiente, fue plantada en el año 2013 con las especies Eucalyptus camaldulensis, Eucalyptus tereticornis Smith y Eucalyptus globulus. Las plantas se produjeron en el vivero del Centro del Secano de

Cuadro 1. Análisis de suelo de la parcela de estudio.

Study plot soil analyses.

\begin{tabular}{cccccccccccccccc}
\hline $\begin{array}{c}\text { Materia } \\
\text { orgánica }\end{array}$ & $\mathrm{pH}$ & $\begin{array}{c}\text { Conductividad } \\
\text { eléctrica }\end{array}$ & $\mathrm{N}$ & $\mathrm{P}$ & $\mathrm{K}$ & $\mathrm{Mn}$ & $\mathrm{Zn}$ & $\mathrm{Cu}$ & $\mathrm{Fe}$ & $\mathrm{B}$ & $\mathrm{Ca}$ & $\mathrm{Mg}$ & $\mathrm{K}$ & $\mathrm{Na}$ \\
\hline$(\%)$ & & $\left.(\mathrm{dS} \mathrm{m})^{-1}\right)$ & & \multicolumn{1}{c}{$\left(\mathrm{mg} \mathrm{kg}^{-1}\right)$} \\
\hline 2,61 & 5,74 & 0,018 & 5 & 5 & 81 & 50,77 & 0,33 & 1,38 & 21,65 & 0,3 & 1,71 & 0,61 & 0,21 & 0,02 \\
\hline
\end{tabular}


la Universidad Católica del Maule, con semilla adquirida al CSIRO Australiano. Las semillas se pusieron a germinar en cámara de germinación previo remojo en ácido giberélico para romper la latencia de las mismas. Las semillas germinadas se prepararon para germinar en contenedores de $140 \mathrm{~mL}$ (Termomatrices ${ }^{\circledR}$, Santiago, Chile) y se cultivaron en un vivero desde mediados de septiembre 2012 hasta mediados de mayo de 2013. Una mezcla de corteza de $P$. radiata y perlita $(7: 3, \mathrm{v} / \mathrm{v})$, más el fertilizante de liberación lenta Basacote ${ }^{\circledR} 9 \mathrm{M}\left(16 \% \mathrm{~N}, 8 \% \mathrm{P}_{2} \mathrm{O}_{5}, 12 \%\right.$ $\mathrm{K}_{2} \mathrm{O}, 12 \% \mathrm{SO}_{3}, 2 \% \mathrm{MgO}, 0,02 \% \mathrm{~B}, 0,05 \% \mathrm{Cu}, 4 \% \mathrm{Fe}$ y $0,06 \% \mathrm{Mn}$ ), a una dosis de $4 \mathrm{~g} \mathrm{~L}^{-1}$ de sustrato, se usó como medio de crecimiento. Las plántulas se protegieron con una malla plástico al $50 \%$ (Raschel ${ }^{\circledR}$ ) y se regaron hasta su capacidad de campo. La plantación se efectuó de forma manual con una pala plantadora y se estableció con un marco de plantación de $3 \mathrm{~m}$ entre filas y un metro entre plantas (i.e., densidad de 3.333 plantas ha ${ }^{-1}$ ). Los tratamientos fueron dos diferentes niveles de manejo, uno básico y uno intensivo. Se establecieron parcelas de $2.500 \mathrm{~m}^{2}$ para cada especie y tratamiento, realizándose tres réplicas de cada tratamiento, teniendo una superficie efectiva de ensayo de $45.000 \mathrm{~m}^{2}\left(2.500 \mathrm{~m}^{2} \times 3\right.$ especies $\times 2$ tratamientos x 3 réplicas). En el tratamiento de manejo intensivo, el suelo fue preparado por medio de un subsolado a una profundidad de $50 \mathrm{~cm}$, seguido de un arado. A continuación, se aplicó herbicida pre-plantación (Terbutilazina, Click ${ }^{\circledR} 75,3 \mathrm{~kg} \mathrm{ha}^{-1}$ ). La plantación fue realizada de forma manual y se hizo una fertilización usando $600 \mathrm{~kg} \mathrm{ha}^{-1}$ de fertilizante con $8 \%$ de nitrógeno, $24 \%$ de fósforo $\left(\mathrm{P}_{2} \mathrm{O}_{5}\right)$ y $24 \%$ de potasio $\left(\mathrm{K}_{2} \mathrm{O}\right)$. Los anteriores tratamientos y dosis fueron elegidos siguiendo las recomendaciones de plantación para este tipo de cultivos en áreas de secano en función de los resultados obtenidos del análisis de suelo. La cosecha de muestras se hizo de forma manual (10 individuos por especie y nivel de manejo, de forma aleatoria en los sectores donde no hubo mortalidad). Para efectos del cálculo del balance energético se simuló el uso de una cosechadoraastilladora-cargadora diseñada y creada por Spapperi ${ }^{\circledR}$ y un remolque llevado por tractor para la recogida del astillado. Lo anterior debido a que a una escala de trabajo normal (más de 10 hectáreas), la cosecha manual supondría un gasto energético, económico y de tiempo muy elevado. En cuanto al manejo básico hubo un subsolado con camellón (20 cm de profundidad). La plantación y cosecha se realizó de la misma forma que en el manejo intensivo. La cosecha se realizó en el año 2016, cuando la plantación contaba con una edad de tres años, en ambos tratamientos.

Biomasa y poder calorifico. A todas las plantas se les midieron las variables morfológicas diámetro a altura de pecho (DAP en $\mathrm{mm}$ ) y altura $(\mathrm{cm})$. El DAP se midió con un pie de metro graduado en milímetros y la altura total se midió mediante un jalón graduado en centímetros, desde la superficie del suelo hasta el ápice central de la planta. Siguiendo la metodología planteada por Nassi o di Nasso et al. (2010), para el cálculo de la biomasa, las muestras, 10 por cada especie y nivel de manejo (60 muestras en total), se tomaron dentro de filas con al menos 10 metros continuos, y consistieron en plantas completas, eligiendo las que crecieron al interior de la plantación, descartando filas externas o próximas a zonas de mucha mortalidad. Las plantas fueron elegidas al azar ya que en cuanto a la variabilidad al interior de los tratamientos de manejo no hubo diferencias significativas para ninguna de las variables (altura, diámetro y biomasa producida) entre las tres especies $(P>0,05)$ Las diferentes muestras fueron introducidas en estufa a $60^{\circ} \mathrm{C}$ hasta que alcanzaron peso constante. Considerando que las plantas que murieron el año uno fueron repuestas, la biomasa por hectárea se obtuvo de la media de los individuos seleccionados multiplicada por la densidad de plantación. De esta forma se podría estimar la biomasa potencial total de acuerdo a la densidad planteada inicialmente, la que se podría obtener con un manejo adecuado de la plantación.

Para el cálculo del poder calorífico se seleccionaron 10 muestras de todos los tejidos del árbol (hojas, ramas y tallo) por cada tratamiento (60 muestras en total) y se obtuvo el poder calorífico usando la bomba calorimétrica de Mahler® según la norma ASTM D2015 (ASTM 1998).

Balance energético. Para la determinación del balance energético se siguió la metodología usada por Cabrera et al. (2014), considerando los flujos de energía asociados a las operaciones necesarias para el cultivo de las diferentes especies, excluyendo la energía necesaria para el transporte del producto a la central de conversión, es decir, se ha realizado un balance energético a pie de campo u orilla del camino. La energía de mano de obra y producción de las plantas para la plantación no se tuvo en cuenta por ser inferior al 0,2\% del total de la energía (Boehmel et al. 2008). En la determinación de los insumos (inputs) energéticos necesarios para la realización del cultivo se consideraron:

- Los costos energéticos para la fabricación y reparación de las máquinas agrícolas.

- Los costos para la producción de los fertilizantes, herbicidas.

- Los costos por el consumo de combustible y aceite lubricante en las diferentes operaciones de cultivo.

Se asumió que las máquinas y los aperos se usaron en 200 hectáreas y tienen una vida útil de 10 años (Bonari et al. 1999). Los costos energéticos para la construcción, depreciación y mantenimiento de los tractores se calcularon teniendo en cuenta la vida media y el tiempo de uso en cada operación de cultivo (Cabrera et al. 2014). La producción de energía obtenida del sistema (outputs) fue determinada multiplicando la producción en materia seca por el poder calorífico de la biomasa de las diferentes especies. Sucesivamente, se calculó la producción de energía neta (output-input). Para el cálculo de la energía neta, se usó la siguiente fórmula [1]: 
Outputs - Inputs $=$ Energía producida (poder calorífico x cosecha) - Energía consumida (operaciones + energía de los factores productivos)

Se creó una base de datos para determinar la energía empleada en la realización de cada una de las operaciones de cultivo, detallando el costo energético directo y el indirecto de las diferentes operaciones, siendo el costo directo el que se refiere al costo de la operación específica, mientras que el costo indirecto es el que se refiere al costo energético debido a la construcción del tractor y de los aperos. Los datos presentados en el cuadro 2 forman parte la base de datos del software Sisco®, desarrollado para el cálculo de balances energéticos en el Centro Enrico Avanzi de Pisa (Bonari 1999).

Análisis estadístico. Se realizó una comparación de medias entre factores y sus interacciones los cuales se analizaron con ANDEVA. Todas las variables, fueron analizadas con el enfoque de modelo lineal general (GLM) para el análisis de varianza. Se utilizó la suma de cuadrados tipo III para la prueba F, que se calculó utilizando el software SPSS versión 18.0. Previo al análisis, se examinaron los supuestos de normalidad y homogeneidad de la varianza. Los términos del modelo se ajustaron de la siguiente forma [2]:

$$
\mathrm{Y}_{\mathrm{ijk}}=\mu+\mathrm{P}_{\mathrm{i}}+\mathrm{E}_{\mathrm{j}}+\left(\mathrm{P}^{*} \mathrm{E}\right)_{\mathrm{ij}}+\mathrm{e}_{\mathrm{ijk}}
$$

donde, $\mathrm{Y}_{\mathrm{ij} \mathrm{k}}$ es la medición fenotípica (diámetro, altura, biomasa), $\mu$ es la media general, $\mathrm{P}_{\mathrm{i}}$ es el efecto fijo del $\mathrm{i}$ ésimo tratamiento de manejo ( $\mathrm{i}=$ intensivo, básico), $\mathrm{E}_{\mathrm{j}}$ es el efecto fijo de la j-ésima especie $(\mathrm{j}=E$. camaldulensis, E. tereticornis, E. globulus), $\mathrm{P}^{*} \mathrm{E}_{\mathrm{j}}$ es la interacción entre el efecto del tratamiento de manejo y la especie, $\mathrm{y} \mathrm{e}_{\mathrm{ij \textrm {k }}}$ es el error aleatorio experimental.

\section{RESULTADOS}

Biomasa y poder calorifico. Los crecimientos en altura fueron diferentes para el tipo de manejo (figura 1), siendo mayores en las especies manejadas con un nivel intensivo. No hubo diferencias significativas en el diámetro en cuanto a diferente tipo de manejo. Respecto a la cantidad de biomasa producida, y como era de esperar, fueron mayores los valores obtenidos en el manejo intensivo respecto al manejo básico (cuadro 3). Por otro lado, no hubo interacción entre los diferentes factores $(P>0,05)$.

En cuanto al poder calorífico, los valores fueron muy similares entre las tres especies. E. camaldulensis tuvo un poder calorífico de 19,2 $\mathrm{MJ} \mathrm{kg}^{-1}$, E. tereticornis de $19,5 \mathrm{MJ} \mathrm{kg}^{-1}$ y E. globulus de $18,1 \mathrm{MJ} \mathrm{kg}^{-1}$.

Balance energético. En cuanto al balance energético, los resultados indicaron que la energía neta producida fue mayor en el manejo intensivo con la especie E. camaldulensis (cuadro 4). En general, si se compara la misma especie con diferentes niveles de manejo, en todos los casos, el manejo intensivo arrojó una producción de energía neta mayor. Sin embargo, al comparar especies, los valores netos más altos

Cuadro 3. Nivel de significancia para los factores y variables morfológicas analizadas (D: diámetro, $\mathrm{H}$ : altura). *Valores estadísticamente significativos $(P<0,05)$.

Level of significance for the factors and morphological variables analyzed (D: diameter, $\mathrm{H}$ : height). *Statistically significant values $(P<0.05)$.

\begin{tabular}{cccc}
\hline Variable & Especie (E) & Manejo $(\mathrm{M})$ & EÍM \\
\hline $\mathrm{D}(\mathrm{cm})$ & 0,34 & 0,07 & 1,00 \\
$\mathrm{H}(\mathrm{m})$ & 0,20 & $0,00^{*}$ & 0,90 \\
Biomasa $\left(\mathrm{Mg} \mathrm{ha}^{-1}\right)$ & 0,13 & $0,01^{*}$ & 0,99 \\
\hline
\end{tabular}

Cuadro 2. Energía directa e indirecta empleada en las operaciones de cultivo, fertilización, herbicidas y cosecha. Direct and indirect energy used in farming operations, fertilizer, herbicides and crop.

\begin{tabular}{lcccc}
\hline & \multicolumn{3}{c}{ Energía consumida $\left(\mathrm{MJ} \mathrm{ha}^{-1}\right)$} \\
\cline { 2 - 4 } \multicolumn{1}{c}{ Operaciones } & Tractor $(\mathrm{kW})$ & Directa & Indirecta & Total \\
\hline Subsolado profundo $(40-50 \mathrm{~cm})$ & 132 & $2.804,8$ & 479 & $3.283,8$ \\
Arado & 73 & 332 & 308 & 640 \\
Aplicación fertilizante & 48 & 334,6 & 161 & 495.6 \\
Fertilizante 8:24:24 $\left(\mathrm{MJ} \mathrm{kg}^{-1}\right)$ & - & 12.7 & & 12,7 \\
Aplicación herbicida & 48 & 303 & 164 & 467 \\
Herbicida Click 75 $\left(\mathrm{MJ} \mathrm{kg}^{-1}\right)$ & - & 91,2 & & 91,2 \\
Plantación & - & 800 & & 810 \\
Cosecha & 132 & 2.198 & 503 & 2.701 \\
\hline
\end{tabular}



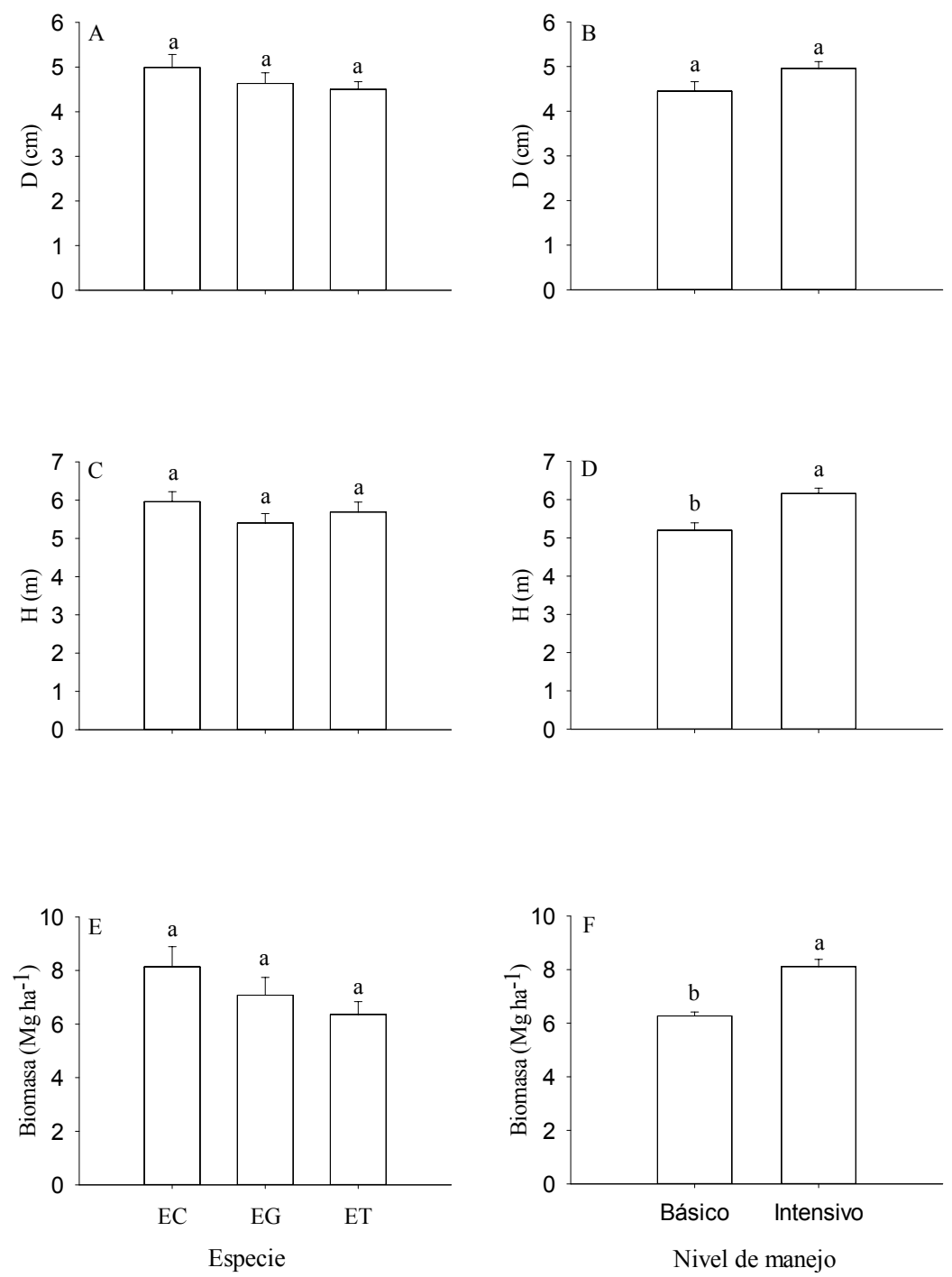

Figura 1. Eucalyptus camaldulensis, E. globulus y E. tereticornis con tres años de edad: A) Diámetro medio. B) Diámetro medio con dos niveles de manejo (básico e intensivo). C) Altura media. D) Altura media con dos niveles de manejo (básico e intensivo). E) Producción de biomasa seca. F) Producción de biomasa media con dos niveles de manejo (básico e intensivo). Letras diferentes implican diferencias significativas $(P \leq 0,05)$.

Eucalyptus camaldulensis, E. globulus and E. tereticornis at three years of age: A) Mean diameter. B) Mean diameter with two levels of management (basic and intensive). C) Average height. D) Average height with two levels of management (basic and intensive). E) Dry biomass production. F) Dry biomass production with two management levels (basic and intensive). Different letters imply significant differences $(P \leq 0.05)$.

Cuadro 4. Energía neta producida y eficiencia energética para tres especies de eucalipto con dos niveles de manejo.

Net energy produced and energy efficiency for three eucalyptus species with two management levels.

\begin{tabular}{clrccc}
\hline Nivel de manejo & \multicolumn{1}{c}{ Especie } & Input $\left(\mathrm{GJ} \mathrm{ha}^{-1}\right)$ & Output $\left(\mathrm{GJ} \mathrm{ha}^{-1}\right)$ & Energía neta $\left(\mathrm{GJ} \mathrm{ha}^{-1}\right)$ & Eficiencia energética \\
\hline \multirow{4}{*}{ Básico } & Eucalyptus camaldulensis & 4,35 & 138,24 & 133,89 & 31,77 \\
& Eucalyptus tereticornis & 4,35 & 107,25 & 102,90 & 24,65 \\
& Eucalyptus globulus & 4,35 & 112,22 & 107,87 & 25,79 \\
\hline \multirow{3}{*}{ Intensivo } & Eucalyptus camaldulensis & 14,67 & 174,72 & 160,05 & 11,91 \\
& Eucalyptus tereticornis & 14,67 & 140,40 & 125,73 & 9,57 \\
& Eucalyptus globulus & 14,67 & 144,80 & 130,13 & 9,87 \\
\hline
\end{tabular}


se obtuvieron con E. camaldulensis, en ambos niveles de manejo. En el caso de la eficiencia energética, fue mucho mejor en el nivel de manejo básico que en el intensivo para todas las especies.

\section{DISCUSIÓN}

Biomasa y poder calorífico. Es ampliamente sabido que las plantaciones de especies del género Eucalyptus tienen el potencial para ser establecidas en ambientes Mediterráneos (Cromer 1976, Cabrera et al. 2014). Los resultados de la presente investigación demuestran que una mayor inversión energética en el manejo se verá reflejada en una mayor producción de biomasa y, por lo tanto, el balance energético será mayor que en el caso de un manejo básico de la plantación.

En relación al desempeño por especie, se puede observar una menor cantidad de biomasa producida por la especie E. globulus respecto a otros ensayos donde las condiciones de sitio y manejo eran mucho mejores (Guo et al. 2006), probablemente debido a la restricción hídrica inherente a los sitios de secano. Sin embargo, si se comparan los resultados con estudios realizados en Australia, donde la mayoría se ha desarrollado en áreas con clima mediterráneo (seis meses de período seco, precipitaciones de $350 \mathrm{~mm}$ año ${ }^{-1}$ ), se aprecian resultados similares, incluso mejores. Por ejemplo, Cromer et al. (1976) encontraron que la biomasa total (densidad de 2.196 plantas ha ${ }^{-1}$ ) varió de $1 \mathrm{Mg} \mathrm{ha}^{-1}$ año ${ }^{-1}$ sin fertilización hasta $9 \mathrm{Mg} \mathrm{ha}^{-1}$ año $^{-1}$ con fertilización (con niveles de nitrógeno de $202 \mathrm{~kg} \mathrm{ha}^{-1} \mathrm{y}$ fósforo de $90 \mathrm{~kg} \mathrm{ha}^{-1}$ ) a los dos años, y 6 y $30 \mathrm{Mg} \mathrm{ha}^{-1} \mathrm{año}^{-1}$ a los cuatro años, sin y con fertilización, respectivamente. También para esta misma especie, Wise y Pitman (1981) reportaron una productividad de 11 a $16 \mathrm{Mg} \mathrm{ha}^{-1}$ año $^{-1}$ a los 10 años, demostrando que los mejores rendimientos se obtienen con altas densidades y rotaciones cortas. El poder calorífico de esta especie varía, de acuerdo a Pérez (2001), en función de la edad, entre valores que van desde las $17,17 \mathrm{MJ} \mathrm{kg}^{-1}$ a los 6-10 años hasta las $17,58 \mathrm{MJ} \mathrm{kg}^{-1} \mathrm{a}$ los 16-20 años. Los valores obtenidos en este estudio (i.e., $18,1 \mathrm{MJ} \mathrm{kg}^{-1}$ a los tres años) son ligeramente mayores a los reportados en la bibliografía. En este sentido, hay que tener especial cuidado con la contaminación con inertes que pueden ingresar durante el proceso de recolección y astillado y que evidentemente reducirán la energía neta disponible. Adicionalmente, la degradación microbiológica producto de almacenamientos prolongados de biomasa en condiciones de humedad ideales para su reproducción, reduce la energía neta disponible, ya que parte será utilizada como alimento por los microorganismos (Jara 2009). Estos hechos podrían explicar las diferencias entre los valores obtenidos en el estudio y los encontrados en la bibliografía.

En cuanto al $E$. camaldulensis, cabe mencionar que es una especie de rápido crecimiento y sus rendimientos en producción de madera son variables y dependen del sitio de plantación. A nivel mundial, en ciertas localidades el crecimiento no supera $12 \mathrm{~m}^{3} \mathrm{ha}^{-1}$ año $^{-1}$, en tanto que, en Chile, los valores van de 8 a $20 \mathrm{~m}^{3} \mathrm{ha}^{-1}$ año $^{-1}$ (INFOR-INDAP-FIA 2000). En el caso de rendimientos para biomasa, en la India, debido a que los terrenos disponibles para los cultivos dendroenergéticos son tierras muy degradadas, las plantaciones de Eucalyptus son manejadas teniendo en consideración mejoramiento genético, fertilización y riego. Los rendimientos alcanzados en E. camaldulensis son de $3 \mathrm{Mg} \mathrm{ha}^{-1}$ año $^{-1}$ de materia seca, aunque con mejoramiento genético y fertilización se puede alanzar las $12 \mathrm{Mg}$

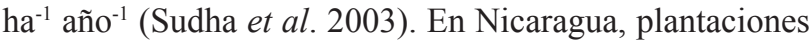
de E. camalulensis establecidas en suelos degradados con $1.900 \mathrm{~mm}^{2}$ añ ${ }^{-1}$ de precipitación, sin mejoramiento genético ni fertilización, rinden del orden de las $13 \mathrm{Mg} \mathrm{ha}^{-1} \mathrm{año}^{-1}$ (Van den Broek y Van Wijk 1998, Van den Broek et al. 2001). Como se puede apreciar, los valores obtenidos en este estudio están dentro del rango de los encontrados en la bibliografía, siendo el manejo intensivo el que produce una mayor cantidad de biomasa. Para la especie se ha reportado un valor de poder calórico de 19,2 $\mathrm{MJ} \mathrm{kg}^{-1}$ (Hobbs et al. 2009), igual al valor obtenido en este estudio.

En lo que respecta a E. tereticornis, es una especie versátil, de crecimiento rápido ampliamente distribuida por el mundo. Ha sido especialmente exitoso en áreas con lluvias de verano seguidas por una estación seca de moderada a severa (trópicos secos estacionalmente), aunque procedencias de rápido crecimiento adaptadas a las lluvias de invierno también están disponibles en la parte sur de su distribución natural. En Australia, la productividad es considerablemente menor que los mejores rendimientos obtenidos en otros países donde, las medias de los incrementos anuales son muy variados dependiendo de la calidad del sitio, las variables climáticas como la precipitación, procedencia usada, carga animal y manejo de plantaciones. Los incrementos medios anuales presentan rangos de alrededor de $30 \mathrm{~m}^{3} \mathrm{ha}^{-1}$ en el Congo (procedencias del norte de Queensland) descendiendo a 19,8-1,3 $\mathrm{m}^{3} \mathrm{ha}^{-1}$ para las plantaciones de 8 años de edad en la India (Tewari 1992). En ensayos con E. tereticornis de 12 años de edad en el noreste de México, se obtuvieron 7,9 $\mathrm{m}^{3} \mathrm{ha}^{-1}$ año ${ }^{-1}$ de madera y $13,7 \mathrm{~m}^{3} \mathrm{ha}^{-1}$ año ${ }^{-1}$ de leña (Foroughbakhch et al. 1997). En otro estudio en la India, con diferentes densidades de plantación y riego, obtuvieron valores de biomasa que oscilaron entre las 5,5 y 26,2 $\mathrm{Mg} \mathrm{ha}^{-1} \mathrm{año}^{-1}$ (Minhas et al. 2015). El poder calorífico de la madera de esta especie presenta valores del orden de las 19,51 $\mathrm{MJ} \mathrm{kg}^{-1}$ (Quirino et al. 2005), valor que coincide con el obtenido en este estudio.

En general, los valores de biomasa obtenidos son bajos si los comparamos con otros trabajos, esto puede deberse a que durante la temporada 2012, previa al establecimiento, las precipitaciones fueron solo de $507 \mathrm{~mm}$. Además, durante la temporada de establecimiento (año 2013), las precipitaciones también fueron menores a la media de un año normal (696-926 mm año $\left.{ }^{-1}\right)$ llegando a los $350 \mathrm{~mm}$. Los mayores crecimientos obtenidos en el tratamiento in- 
tensivo son esperados ya que las condiciones generadas a la planta son más favorables que en el tratamiento básico. El uso de fertilizantes al establecimiento de plantaciones manejadas intensivamente, partiendo de un adecuado control de malezas, es una herramienta clave para el aumento de la productividad forestal de especies de rápido crecimiento en Chile (Rubilar et al. 2008). Sin embargo, es necesario precisar cuál de las medidas de mejoramiento es más efectiva, ojalá apoyado por un diagnóstico preciso de qué factores del suelo (físicos y nutritivos) y clima son determinantes en el crecimiento de las especies en cuestión, para el sitio en que se desee cultivarlas.

Balance energético. En todos los supuestos, el balance de energía es positivo, sin embargo, la cantidad de energía potencialmente producida es mayor en el manejo intensivo, aunque la eficiencia energética es mejor en el manejo básico para todas las especies. En la zona mediterránea de Chile, con suelos y clima en general favorables para los eucaliptos seleccionados, es probable que en la primera rotación se hubiera logrado este resultado. Pero, precisando los factores limitantes y ejecutando las mejoras correspondientes, puede lograrse un resultado más eficiente del cultivo. Es importante señalar que el cultivo intensivo de especie forestales de rápido crecimiento, con rotaciones muy cortas, son extremadamente extractivas en nutrientes, con demandas similares a cultivos agrícolas y/o plantaciones tradicionales en fase de crecimiento acelerado. Es por lo anterior que se requeriría de una reingeniería de silvicultura específica de sitio, y se podría considerar el posible uso de especies fijadoras de nitrógeno y/o rotaciones de especies en este tipo de cultivos dendroenergéticos.

Considerando que el área donde se ha desarrollado el estudio es una zona donde la tenencia de la tierra corresponde generalmente a pequeños $\mathrm{y} / \mathrm{o}$ medianos propietarios que no cuentan con gran cantidad de recursos (especialmente financieros), para ellos, la mejor alternativa sería la de un manejo básico. Faúndez (2003), en un estudio donde compara la misma área de cultivo (10 ha) en diversos regímenes de manejo y con la especie E. camaldulensis, concluye que el costo potencial mínimo de producir energía, utilizando regímenes de cultivo no intensivo es como máximo la mitad del costo potencial de los intensivos. Este hecho es alentador, dado la gran área de tierra disponible en el centro de Chile para la silvicultura no intensiva en comparación con la disponible para la silvicultura intensiva.

En bibliografía no se han encontrado trabajos que utilicen esta misma metodología en el cálculo del balance energético de especies del género Eucalyptus. Sin embargo, sí existen reportes respecto del cálculo del balance energético de Populus spp. y, en este sentido, los resultados obtenidos en esta investigación son comparables con los extraídos en otros estudios (Matthews 2001), que muestran que el cultivo de Populus deltoides Marsh. tiene un balance energético positivo. Otros estudios (Matthews 2001, Nonhebel 2002) también tuvieron un balance ener- gético positivo en el cultivo de Populus spp. En ambos casos, el balance neto tuvo una mayor cantidad de energía ya que los estudios se realizaron en condiciones de sitio mejores que las del presente trabajo.

En general, estos resultados confirman la hipótesis planteada, es decir, una mayor inversión energética en el manejo ha servido para superar algunas limitaciones de sitio (fertilidad y compactación), lo que se ve reflejado en una mayor producción de biomasa. Por lo tanto, el balance energético ha sido más positivo en el manejo intensivo que en el caso de un manejo básico de la plantación.

\section{CONCLUSIONES}

El cultivo de especies de Eucalyptus adaptadas a zonas de secano puede ser una alternativa viable como fuente de energía renovable, especialmente la especie Eucalyptus camaldulensis. Un nivel de manejo intensivo se traduce en una producción de energía mayor, aunque la eficiencia energética es mejor en el nivel de manejo básico para todas las especies. A futuro, se deben realizar estudios más precisos para determinar que factor es el determinante al manejar el suelo y su fertilidad sobre la productividad, así como medidas de conservación para favorecer la infiltración de agua en el suelo, manejar los excedentes y evitar así la erosión. También se debe incluir el transporte de la biomasa a la central y el rendimiento de esta última, además se deben incluir cosechas posteriores, de manera de incorporar la capacidad de rebrote en el balance energético y en la decisión de la especie a elegir para tener una visión global del balance energético de estas especies en zonas de secano.

\section{REFERENCIAS}

ASTM (American Society for Testing and Materials, US). 1998. ASTM Standards D2015-96: Standard test method for gross calorific value of coal and coke by the adiabatic bomb calorimeter. In American Society for Testing and Materials. Annual Book of ASTM Standards, Section 5, Vol. 05.05. West Conshohocken, PA., USA. p. 239-247.

Boehmel C, I Lewandowski, W Claupei. 2008. Comparing annual and perennial energy cropping systems with different management intensities. Agricultural Systems 96: 224-236. DOI: 10.1016/j.agsy.2007.08.004

Bonari E, A Peruzzi, M Mazzoncini, N Silvestri. 1992. Valutazione energetiche di sistema produttivi a diverso livello di intensificazione colturale. L'Informatore Agrario 1: 11-25.

Bonari E, N Silvestri, S Pampana. 1999. Sisco: a practical tool to assess alternative cropping systems' performances. In Proceedings International Symposium Modelling Cropping Systems, Lleida España. p. 285-286.

Cabrera AM, C Tozzini, S Espinoza, R Santelices, E Bonari. 2014. Cálculo del balance energético de una plantación de Populus deltoides clon Lux con fines energéticos en un sitio con ambiente mediterráneo. Bosque 35(2): 133-139. DOI: 10.4067/S0717-92002014000200001

Casanova M, W Lucio, R Maldonado. 2007. Correlación entre 
World Reference Base y Soil Taxonomy para los suelos de la VII región del Maule de Chile. Revista de la Ciencia del Suelo y Nutrición Vegetal 7(2): 14-21. DOI: $10.4067 /$ $\underline{\text { S0718-27912007000200002 }}$

Cromer RN, M Raupach, ARP Clarke, JN Cameron. 1976. Eucalypt plantations in Australia - the potential for intensive production and utilization. In College of Life Sciences and Agriculture, University of Maine. OSLO Biomass Studies. Orono, Australia. p. 29-40.

Faúndez P. 2003. Potential costs of four short-rotation silvicultural regimes used for the production of energy. Biomass and Bioenergy 24(4-5): 373-380. DOI: 10.1016/S09619534(02)00164-2

Foroughbakhch R, LA Háuad, MH Badii, PR Earl. 1997. Establishment and growth of five Eucalyptus species in north-eastern Mexico. Forest, Farm, and Community Tree Research Reports 2:46-50.

Hobbs TJ, M Bennell, J Bartle. 2009. Developing Species for Woody Biomass Crops in lower rainfall southern Australia. FloraSearch 3a. Report to the Joint Venture Agroforestry Program (JVAP) and Future Farm Industries CRC. Publication No. 09/043. 242 p.

INFOR (Instituto Forestal, CL). 2017. Anuario forestal 2017. Santiago, Chile. 188 p. (Boletín estadístico $N^{\circ} 159$ ).

INFOR-INDAP-FIA (Instituto Forestal-Instituto de Desarrollo Agropecuario, Fundación para la Innovación Agraria, CL). 2000. Eucalyptus camaldulensis en Chile: Silvicultura, manejo, productividad y rentabilidad. $62 \mathrm{p}$.

Jara H. 2009. Biomasa y sus propiedades como combustible. Revista Celulosa y Papel. Octubre 2009: 24-28

Matthews RW. 2001. Modelling of energy and carbon budgets of wood fuel coppice systems. Biomass and Bioenergy 21: 1-19. DOI: 10.1016/S0961-9534(01)00016-2

Minhas P. R Yadav, K Lal, R Chaturvedi. 2015. Effect of longterm irrigation with wastewater on growth, biomass production and water use by Eucalyptus (Eucalyptus tereticornis Sm.) planted at variable stocking density. Agricultural Water Management 52: 151-160. DOI: 10.1016/j. agwat.2015.01.009

Nonhebel S. 2002. Energy yields in intensive and extensive biomass systems. Biomass and Bioenergy 22: 159-67. DOI: 10.1016/S0961-9534(01)00071-X

Nassi o Di Nasso N, W Guidi, G Ragaglini, C Tozzini, E Bonari. 2010. Biomass production and energy balance of a 12-year old short-rotation coppice poplar stand under different cutting cycles. GCB Bioenergy 2:89-97. DOI: 10.1111/j.17571707.2010.01043.x

Novoa R. 1989. Potencialidad y limitaciones de la agricultura del secano interior chileno. In Riquelme H, J Sotomayor eds. Seminario realidad y perspectivas agropecuarias del secano interior. Serie Quilamapu No18. Cauquenes, Chile. Instituto de Investigaciones Agropecuarias, Subestación Experimental Cauquenes. p. 9-24.

Guo L, R Sims, D Horne. 2006. Biomass production and nutrient cycling in Eucalyptus short rotation energy forests in New Zealand. II. Litter fall and nutrient return. Biomass and Bioenergy 30: 393-404. DOI: 10.1016/j.biombioe.2005.11.017

Pérez H. 2001. Estudio tecnológico de la madera del eucalipto en Cajamarca con fines estructurales. In I Congreso Nacional del Eucalipto, Facultad de Ciencias Forestales y del Ambiente de la Universidad Nacional del Centro del Perú (UNDCP).

Quirino WF, DO Vale, AT Abreu de Andrade, AP Silva, VL Dos Santos. 2005. Poder calorífico da madeira e de materiais lignocelulósicos. Revista da Madeira 89: 100-106.

Rubilar R, T Fox, L Alle, T Albaugh, C Carlson. 2008. Manejo intensivo al establecimiento de plantaciones forestales de Pinus sp. y Eucalyptus sp. en Chile y Argentina. Informaciones Agronómicas del Cono Sur 40: 1-6.

Santelices R, S Espinoza, A Cabrera. 2015. Especies del género Eucalyptus con potencial energético para ser establecidas en el secano Mediterráneo de la Región del Maule. Talca, Chile. Centro de Desarrollo para el Secano Interior, Universidad Católica del Maule. 64 p.

Scholz V, R Ellerbrock. 2002. The growth productivity, and environmental impact of the cultivation of energy crops on sandy soil in Germany. Biomass and Bioenergy 23: 81-92. DOI: $10.1016 / \mathrm{S} 0961-9534(02) 00036-3$

Sevigne E, CM Gasol, F Brun, L Rovira, JM Pagés, F Camps, J Rieredevall, X Gabarrell X. 2011. Water and energy consumption of Populus spp. bioenergy systems: A case study in Southern Europe. Renewable \& Sustainable Energy Reviews 15: 1133-1140. DOI: 10.1016/j.rser.2010.11.034

Sudha P, H Somashekhar, S Rao, N Ravindranath. 2003. Sustainable biomass production for energy in India. Biomass and Bioenergy 25: 501-515. DOI: 10.1016/S09619534(03)00085-0

Tewari DN, 1992. Monograph on Eucalyptus. Dehra Dun, India. Surya Publications. 361 p.

Van Den Broek R, A Van Wijk. 1998. Generation of electricity with eucalyptus and bagasse in sugar mills in Nicaragua: final report. Rome, Italy. FAO, Utrecht University, Department of Science, Technology and Society.

Van Den Broek R, L Vleeshouwers, M Hoogwijk, A Van Wijkc, W Turkenburga. 2001. The energy crop growth model SILVA: description and application to eucalyptus plantations in Nicaragua. Biomass and Bioenergy 21: 335-349. DOI: 10.1016/S0961-9534(01)00032-0

Wise P, M Pitman. 1981. Nutrient removal and replacement associated with short-rotation eucalypt plantations. Australian Forestry 44(3): 142-152. DOI: $\underline{10.1080 / 00049158.1981 .10674307}$ 